\title{
Assessing Metacognition and Impulsivity in Parkinson's Disease patients with Gambling Behavior
}

\author{
Balconi Michela ${ }^{1,2}$, Angioletti Laura ${ }^{1,2}$
}

${ }^{1}$ Research Unit in Affective and Social Neuroscience, Catholic University of the Sacred Heart, Milan, Italy ${ }^{2}$ Department of Psychology, Catholic University of the Sacred Heart, Milan, Italy

\section{OBJECTIVES}

To investigate impulsivity levels in subgroups of Parkinson's Disease (PD) patients with different gambling status, and to test self-awareness of decision-making strategies adopted during lowa Gambling Task (IGT) in this clinical population

\section{BACKGROUND and AIMS}

Impaired metacognition skills and impulsivity are critical factors specifically in gambling behavior subsistence.

Our aim was to differentiate between PD patients (with and without active gambling behavior), showing not only vulnerable individual differences but also a reduced insight and metacognition about their gambling behavior, in order to develop tailored clinical interventions targeting impaired these metacognitive abilities.

\section{MATERIALS and METHOD}

Forty-eight PD participants ( 40 males, $M_{a g e}=62,95 \quad S D=7,72$ ) were divided into three subgroups: PD Gamblers (PDG), PD patients with a previous history of gambling (PD Non-Gamblers, PDNG) and PD controls. For details see Table (Mean (SD), raw scores are reported). Impulsivity was assessed by Barratt Impulsiveness Scale (BIS-11) [1,2]. After performing IGT [3], patients filled in a self-report measure to assess metacognitive strategies adopted during the task.

\section{RESULTS}

\section{Metacognitive post-experiment questionnaire $[4,5,6]$}

Composed of 4 aspects covered by 4 items:

Item 1.the general use of a planned strategy metacognition of planning:

"Were you able to apply a strategic plan during the game?"

IItem 2.the awareness of applying a strategy during the task metacognition on the self-awareness:

| "Were you aware of using a strategy during the game?"

Item 3.the change of strategy across the trials metacognition about the flexible strategy:

"Did you change your strategy during the game?"

Item 4.the sensation of having applied a functional strategy metacognition of efficacy:

"Do you think you used an efficacious strategy?"

IFor BIS-11: ANOVA revealed a significant main effect for Group factor for BIS-11 total score $(F[2,41]=3.82, p=.030)$. Pairwise comparison -revealed that PDG group scored higher at BIS-11 total score than $C G(F[1,41]=4.89, p=.010)$ (Figure 1a).

iSimilarly, a significant main effect was found for Group factor for BIS Motor Impulsivity subscale ( $F[2,41]=4.97, p=.012)$. Pairwise comparison revealed that PDG group scored higher at this subscale than the CG group (Figure 1b).

About IGT, Group factor showed significant main effect $(F[2,41]=8.45, p \leq .001, \eta 2=.38)$. Indeed post-hoc paired comparisons revealed lmore negative IGT values (more disadvantageous than advantageous choices) for PDG than PDRG (F[1, 41]=8.11, $\mathrm{p} \leq .001, \eta 2=.38)$ and: CG $(F[1,41]=8.53, p \leq .001, \eta 2=.39)$. Similarly significant differences were found between $P D R G$ and $C G(F[1,41]=7.03, p \leq .001, \eta 2=;$ I.33), with more negative IGT values for PDRG (Figure 2).

Regarding metacognitive measure: Chi-Square analysis revealed a significant difference for item 2 ("Were you aware of using a strategy: during the game?") $(X 2(2,41)=6.12, p=.029)$ : both PDG and CG reported to be aware of their strategy compared to PDRG group. In :addition, significant differences were found between groups for item 4 ("Do you think you used an efficacious strategy?") $(x 2(2,41)=7.07, p=$ : .029). PDG group and CG scored similarly for item 4, where both groups referred to use efficacious strategies in comparison to PDRG.

No other significant differences were found for item $1(x 2(2,41)=1.96, p=.375)$ and item $3(x 2(2,41)=5.11, p=.078)$.

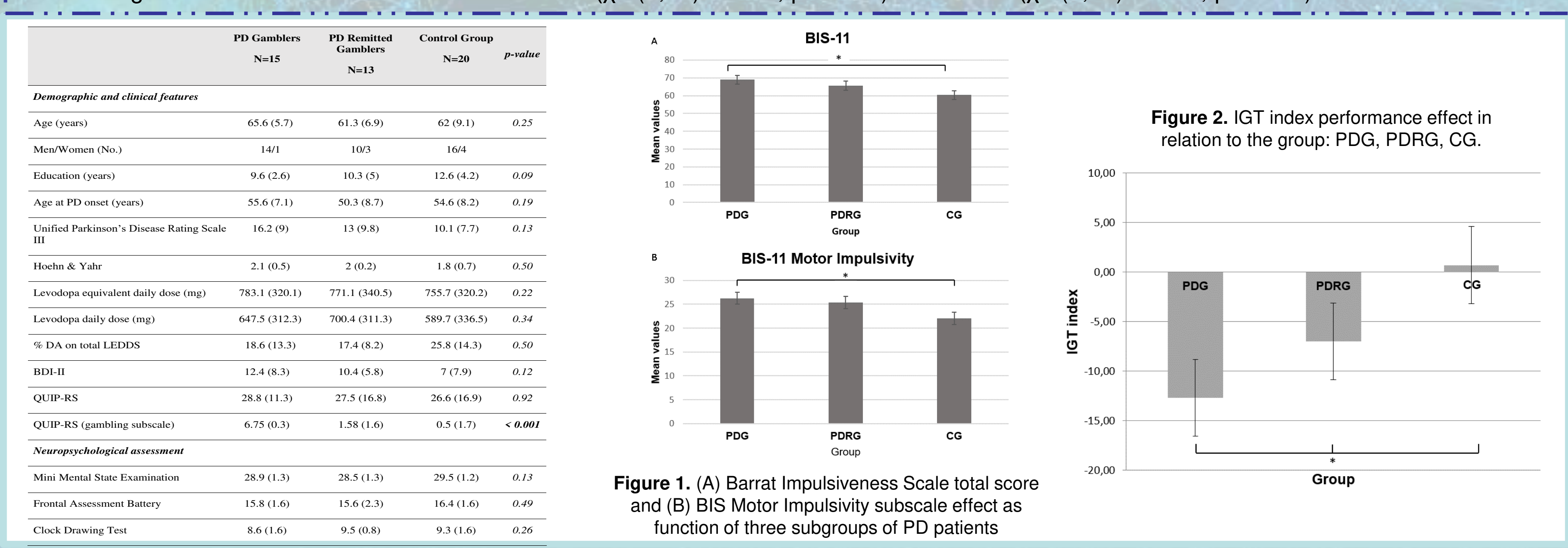

\section{CONCLUSIONS}

BIS-11 and Motor Impulsivity subscale scores were higher in PDG patients than PDNG and controls. IGT behavioral performance reflected more inefficacious decision-making strategies in PDG group, with a decreasing gradient of impairment in the other two groups. This decision-making impairment was not followed by an aware judgment on PDG group behavioral performance, that is the PDG group stated to use an efficacious and aware strategy during the task even if concluding IGT with a negative balance, accordingly, exhibiting an erroneous metacognitive representation.

\section{REFERENCES}

[1] Barratt, E. S. (1985). Impulsiveness Subtraits Arousal [5] Balconi, Michela, Finocchiaro, R., \& Canavesio, Y. $\begin{array}{lll}\text { and Information Processing. In J. Spence \& C. Izard } & \text { (2015a). Left hemispheric imbalance and reward }\end{array}$ (Eds.), Motivation, emotion, and personality (pp. 137-146). mechanisms affect gamblimg behave and: The contribution of
North-Holland: Elsevier Science.
the metacognition and cortical brain oscillations. Clinical 2] Patton, J. (1995). Factor Structure of the Barrat EEG and Neuroscience, 46(3), 197-207. 768-774.
(2015b). Reward Sensitivity (Behavioral Activation [3] Bechara, A., Damasio, A. R., Damasio, H., \& Anderson, System), Cognitive, and Metacognitive Control in following damage to human prefrontal cortex. Cognition, Feedback-Related Negativity, and P300 Effect. The 50(1), 7-15. Journal of Neuropsychiatry and Clinical Neurosciences, [4] Balconi, M, Finocchiaro, R., \& Campanella, S. (2014). 27(3), 219-227. Reward sensitivity, decisional bias, and metacognitive deficits in cocaine drug addiction. Journal of Addiction 